ARTICLE

\title{
Universal three-dimensional crosslinker for all-photopatterned electronics
}

Min Je Kim ${ }^{1,7}$, Myeongjae Lee (10) ${ }^{2,7}$, Honggi Min ${ }^{3}$, Seunghan $\mathrm{Kim}^{4}$, Jeehye Yang ${ }^{4}$, Hyukmin Kweon ${ }^{5}$, Wooseop Lee ${ }^{3}$, Do Hwan Kim (ib ${ }^{5}$, Jong-Ho Choi ${ }^{2}$, Du Yeol Ryu ${ }^{3}$, Moon Sung Kang (i] ${ }^{4 凶}$, BongSoo Kim ${ }^{6 凶} \&$ Jeong Ho Cho ${ }^{3 凶}$

All-solution processing of large-area organic electronics requires multiple steps of patterning and stacking of various device components. Here, we report the fabrication of highly integrated arrays of polymer thin-film transistors and logic gates entirely through a series of solution processes. The fabrication is done using a three-dimensional crosslinker in tetrahedral geometry containing four photocrosslinkable azide moieties, referred to as $4 \mathrm{Bx} .4 \mathrm{Bx}$ can be mixed with a variety of solution-processable electronic materials (polymer semiconductors, polymer insulators, and metal nanoparticles) and generate crosslinked network under exposure to UV. Fully crosslinked network film can be formed even at an unprecedentedly small loading, which enables preserving the inherent electrical and structural characteristics of host material. Because the crosslinked electronic component layers are strongly resistant to chemical solvents, micropatterning the layers at high resolution as well as stacking the layers on top of each other by series of solution processing steps is possible.

\footnotetext{
${ }^{1}$ SKKU Advanced Institute of Nanotechnology (SAINT), Sungkyunkwan University (SKKU), Suwon 16419, Republic of Korea. ${ }^{2}$ Department of Chemistry, Korea University, Seoul 02841, Republic of Korea. ${ }^{3}$ Department of Chemical and Biomolecular Engineering, Yonsei University, Seoul 03722, Republic of Korea. ${ }^{4}$ Department of Chemical and Biomolecular Engineering, Sogang University, Seoul 04107, Republic of Korea. ${ }^{5}$ Department of Chemical Engineering, Hanyang University, Seoul 04763, Republic of Korea. ${ }^{6}$ Department of Chemistry, Ulsan National Institute of Science and Technology (UNIST), Ulsan 44919, Republic of Korea. ${ }^{7}$ These authors contributed equally: Min Je Kim, Myeongjae Lee. ${ }^{凶}$ email: kangms@sogang.ac.kr; bongsoo@unist.ac.kr; jhcho94@yonsei.ac.kr
} 
S olution-processed electronics have been considered a disruptive technology rooted in cost-effective manufacturing ${ }^{1-8}$. The performance of electronic devices based on various solution-processable materials, such as polymer semiconductors, organic small molecules, and nanostructured materials, is being improved continually $y^{6,9-15}$. However, fabrication of these devices entirely through a series of solution processes remains highly challenging, as they comprise a stack of fully patterned electronic components, such as electrode layers, charge transporting interlayers, active channel layers, and insulating layers. While various processing methods have been developed for patterning and stacking each of these electronic component layers separately ${ }^{16-18}$, employing multiple techniques/equipment tailored to form respective layers for fabricating devices entirely through a series of solution processes is nontrivial and cost-ineffective. Moreover, new issues arise when a solution-processed layer is exposed to chemicals during the subsequent processing steps. For instance, conventional photolithography requires multiple steps that involve exposure of a given electronic layer to various chemicals, such as photoresist, developer, and etchant ${ }^{7,16,19}$. This means that finding an appropriate orthogonal chemicals that do not damage the underlying electronic layer is critical for successful pattern formation by photolithography ${ }^{17,20}$. The wider spread of this technique for patterning layers of solution-processable materials is thus limited. Inkjet printing and nanoimprinting methods face several difficulties in producing uniform and large-area electronic devices on a large scale ${ }^{21-26}$. They also suffer from the same drawbacks of the solution-processed materials arising from their poor chemical robustness; the prepatterned underlayer is susceptible to the solution-based deposition process of the layer above it. This leads to degradation in the device performance with poor reliability and reproducibility, which is a major obstacle in realizing all-solutionprocessed electronic devices.

Application of a direct photocrosslinking process to solutionprocessable materials can be a clever strategy for achieving this goal. Selective exposure to a light source enables patterning of the materials. If the crosslinking density within the materials is high enough, the resulting patterns of the materials would become chemically robust. This would enable stacking of patterns on top of other patterns. Moreover, the thermal stability of the materials would be enhanced because of their crosslinked structure. Accordingly, photocrosslinking agents, such as bifunctional organic azides, have been developed ${ }^{27-29}$, and electronic devices employing these layers have been demonstrated ${ }^{6,30-37}$. To the best of our knowledge, however, patterning and stacking of multiple functional materials by consecutive application of photocrosslinking processes have not yet been attempted. An important remark is that because the crosslinking efficiency per molecule of bifunctional organic azides is as low as $36 \%{ }^{38}$, the addition of a large amount of a crosslinking agent is needed to obtain the desired crosslinked structure of the host polymer layer ${ }^{31,33-36}$. In most cases, the use of such a large amount of crosslinking agent leads to a morphological change in the film and deterioration in the electrical and optoelectronic properties of the material. Design of a highly efficient photocrosslinker that can be applied to the patterning process of various solutionprocessable materials with use of a very low amount is thus highly desirable.

Here, we report a simple processing method for fabricating thin-film patterns of solution-processable semiconductors, gate insulators, and conductors using a highly efficient crosslinking agent that does not yield degradation in the electrical properties of the host materials. A crosslinker, referred to as $4 \mathrm{Bx}$, is structured in three-dimensional tetrahedral geometry containing four photocrosslinkable azide moieties, each of which is placed at the four corners of the molecule. $4 \mathrm{Bx}$ is universally applicable to a variety of solution-processable materials containing $\mathrm{C}-\mathrm{H}$ bonds, including polymer semiconductors, polymeric gate dielectrics, and even conductive metal nanoparticles (NPs) bearing organic surfactants in creating crosslinked network. The given host materials can be successfully photopatterned using a small amount of $4 \mathrm{Bx}$ (only $1 \mathrm{wt} \%$ for polymer semiconductors and $5 \mathrm{wt}$ $\%$ for polymeric gate dielectrics and metal NPs). Moreover, fully patterned polymer thin-film transistors (PTFTs) comprising a stack of these materials are fabricated by all-solution processing. The PTFTs based on photocrosslinked polymer films show equivalent performance and better stability compared with those prepared using polymer films without the crosslinker. Furthermore, we demonstrate the fabrication of NOT, NAND, and NOR logic gates based on arrays of all-photopatterned $p$-type and n-type PTFTs.

\section{Results}

Fabrication of all-photopatterned PTFTs and logic circuits. The schematic drawing of the all-photopatterned PTFTs and logic circuits fabricated on a plastic substrate and the photographic images of the resulting devices are show in Fig. 1a. Chemical structures of the materials used are shown in Fig. 1b. Each device contains a stack composed of Ag NP source (S)/drain (D) electrodes, $p$-type poly $((E)-2,5$-bis(2-decyltetradecyl)-3-(thiophen-2yl)-6-(5'-(2-(thiophen-2-yl)vinyl)-[2,2'-bithiophen]-5-yl)pyrrolo $[3,4-c]$ pyrrole-1,4(2H,5H)-dione) (P(DPP2DT-TVT)) or $n$-type poly(4-([2,2'-biselenophen]-5-yl)-2,7-bis(3-octyltridecyl)benzo $[\operatorname{lmn}][3,8]$ phenanthroline-1,3,6,8 $(2 H, 7 H)$-tetraone) (P(NDI3OTSe2)) semiconducting channel material, a poly(methyl methacrylate) (PMMA) gate dielectric, and a $\mathrm{Ag} \mathrm{NP}$ gate electrode. Polystyrene (PS) and poly(vinylidene fluoride-co-hexafluoropropylene) (PVDF-HFP) can also be used for the gate dielectric material (see below). Each of these device component layers was photopatterned using a blend solution of the corresponding electronic material and the (2,2-bis(((4-azido2,3,5,6-tetrafluorobenzoyl)oxy)methyl)propane-1,3-diyl bis(4azido-2,3,5,6-tetrafluorobenzoate), i.e., $4 \mathrm{Bx}$. $4 \mathrm{Bx}$ is structured in three-dimensional tetrahedral geometry containing four photocrosslinkable azide moieties each at the four corners of the molecule. The four azide groups can be activated to generate reactive singlet nitrenes under $254 \mathrm{~nm}$ light; the reactive nitrene can undergo the $\mathrm{C}-\mathrm{H}$ insertion reaction with neighboring alkyl chains to form crosslinked films of the functional materials (see below for further description). Because photocrosslinking reaction can undergo with materials containing alkyl groups, i.e., $\mathrm{C}-\mathrm{H}$ bonds, $4 \mathrm{Bx}$ can be universally applicable to a variety of electronic materials that are processable with common organic solvents; the electronic materials include polymer semiconductors, polymeric gate dielectrics, and even conductive metal NPs bearing organic surfactants. Since the $4 \mathrm{Bx}$ molecule has four crosslinkable units, its crosslinking efficiency, defined as the probability of a single crosslinker molecule to successfully undergo the intended $\mathrm{C}-\mathrm{H}$ insertion reaction, is higher than that of conventional crosslinker with two crosslinkable units. Therefore, only a smaller loading of $4 \mathrm{Bx}$ is needed to form crosslinked network of the host material when it is exploited in the photopatterning process (see below for more details) 33,35 . Synthetic details of $4 \mathrm{Bx}$ and the polymer semiconductors are provided in Supplementary Methods (Supplementary Figs. 1-21).

The schematic fabrication procedure of $p$ - and $n$-type PTFTs by the all-photopatterning process using $4 \mathrm{Bx}$ is summarized in Fig. 1c. Typical PTFTs were fabricated in a top-gate bottomcontact (TGBC) configuration on a polyethylene naphthalate (PEN) substrate. The conducting electrodes, semiconducting channel materials, and polymer gate dielectrics were sequentially 
a

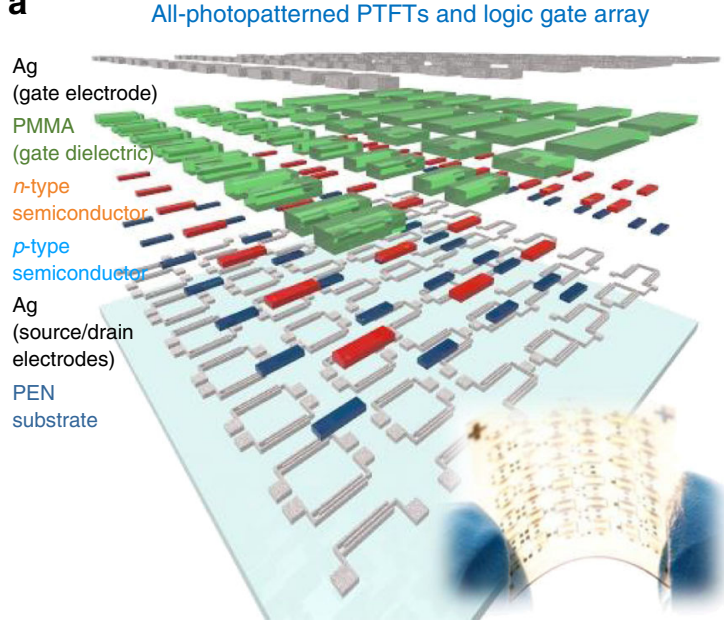

b Electronic materials for PTFTs and logic gates

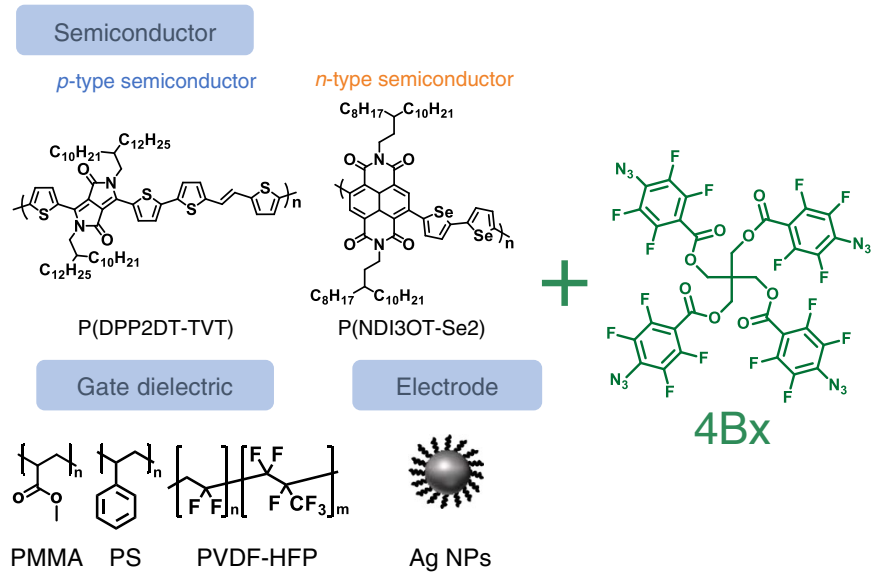

C

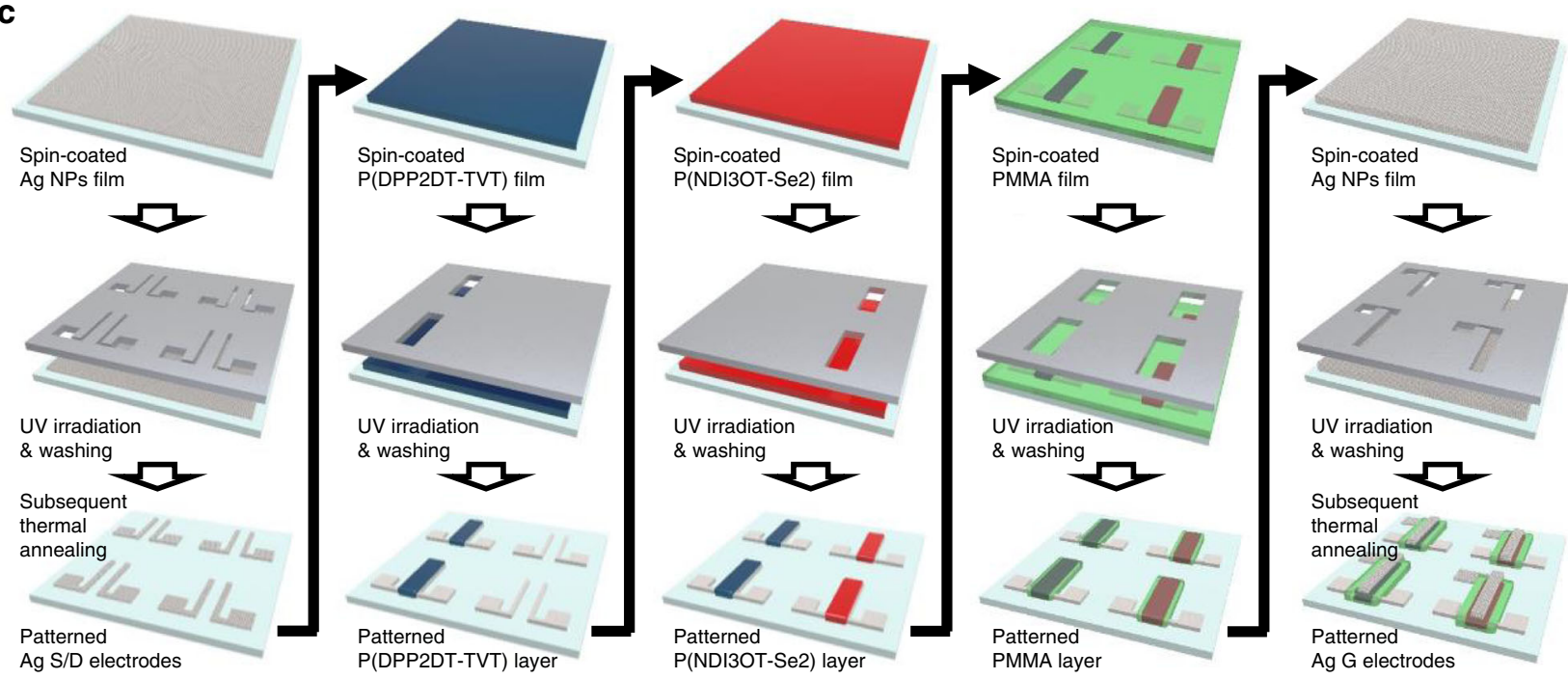

d

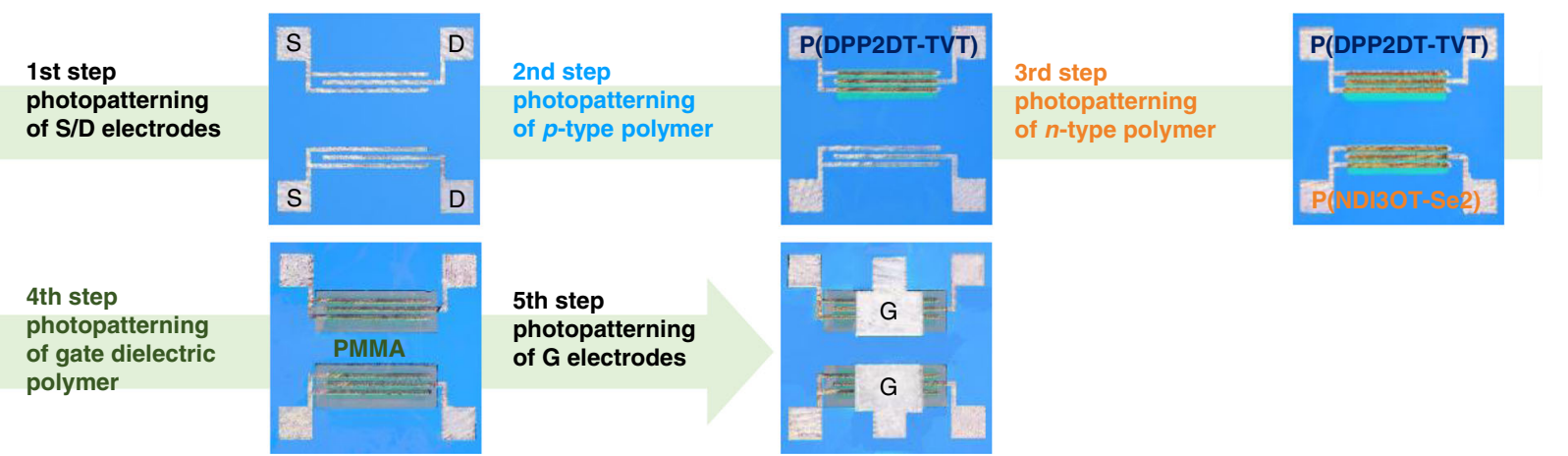

Fig. 1 All-solution-processed, all-photopatterned PTFTs and logic circuits. a Schematic drawing and photographic image of all-photopatterned PTFTs and logic circuits fabricated on a plastic substrate by patterning of semiconducting channel, gate dielectric, and electrode materials. b Chemical structures of electronic materials used in this study. c Schematic description of the fabrication processes for $p$ - and $n$-type PTFTs through all-photopatterning using 4Bx. d Optical microscopy images of the component layers for a PTFT taken after each patterning step.

photopatterned as follows. The first step was the patterning of the Ag NP S/D electrodes. This was done by spin-coating a mixture solution of Ag NPs and 4Bx onto a PEN substrate, followed by selective exposure to UV light $(254 \mathrm{~nm})$ through a photomask. The detail photocrosslinking mechanism of Ag NPs is described below. The uncrosslinked regions were removed simply by the solvent used to coat the Ag NPs and $4 \mathrm{Bx}$ mixture, and the resulting photopatterned $\mathrm{Ag} \mathrm{NP}$ film was then thermally annealed. The annealing process was necessary to impart metallic conductivity to the Ag NP patterns. We note that this two-step process, i.e., photopatterning and thermal annealing, allows us to achieve a high resolution patterning of conductive Ag NP patterns. The second step was the patterning of the $p$-type polymer film, which was achieved by spin-casting a mixture solution of the p-type polymer and $4 \mathrm{Bx}$, exposing the desired area to UV through a photomask, and then washing out the 
uncrosslinked polymer regions. As long as a solvent can dissolve the pristine semiconducting polymer well, it could serve as a good developing solvent (Supplementary Fig. 22). The third step was the photopatterning of the $n$-type polymer film by the same method as that was used in the second step for patterning the $p$ type polymer film. The consecutive application of photopatterning process was only possible due to the structural robustness of the underlying crosslinked $p$-type polymer film. The fourth step was the photopatterning of the polymer gate dielectric layer in the same way by spin-coating of a mixture solution of PMMA insulator and $4 \mathrm{Bx}$. In the fifth step, $\mathrm{Ag} \mathrm{NP}$ films were again photopatterned onto the $p$ - and $n$-channel regions. Optical microscopy images of the patterned PTFT device layers after each photopatterning step are shown in Fig. 1d. We found that all the crosslinked thin films showed improved chemical resistance, and thus, the solvent used in each step for both spin-coating and washing did not damage the underlying crosslinked layer. This implies that stacking and patterning multiple layers of functional materials is possible by the described steps. We emphasize that our study demonstrates the fabrication of all-photopatterned, all-solution-processed PTFTs, which was achieved following a single processing protocol i.e., the photopatterning based on $4 \mathrm{Bx}$ for different functional components of devices.

Characterization of crosslinked and photopatterned electronic components. Figure $2 \mathrm{a}, \mathrm{b}$ shows a schematic illustration of semiconducting polymer film photocrosslinked by $4 \mathrm{Bx}$ and the underlying chemical reaction. When a semiconducting polymer film containing $4 \mathrm{Bx}$ is exposed to UV light, the photolysis of the azide groups in $4 \mathrm{Bx}$ generates highly reactive singlet nitrene $\left({ }^{1} \mathrm{~N}\right)$ along with the evolution of inert nitrogen gas $^{33,35,38,39}$. The dominant reaction between the semiconducting polymer and $4 \mathrm{Bx}$ is the insertion of the singlet nitrene into the $\mathrm{C}-\mathrm{H}$ bond within the alkyl chains of the neighboring polymers ${ }^{35,38}$, which results in the three-dimensionally networked structure. The photochemical reaction of the $4 \mathrm{Bx}$ crosslinker inside the $\mathrm{P}(\mathrm{DPP} 2 \mathrm{DT}-\mathrm{TVT})$ and $\mathrm{P}(\mathrm{NDI} 3 \mathrm{OT}-\mathrm{Se} 2)$ films was confirmed by Fourier transform infrared (FTIR) spectroscopy before and after the films were exposed to UV light, as shown in Fig. 2c and Supplementary Fig. 23a, respectively. The characteristic vibration peak of the azide groups in $4 \mathrm{Bx}$ at $2125 \mathrm{~cm}^{-1}$ disappeared entirely after the films were exposed to UV. The distributions of the photocrosslinked positions could be identified by time-of-flight secondary ion mass spectrometry (TOF-SIMS). The depth profiles were obtained as a function of sputtering time for photocrosslinked polymer films coated on Si wafers. For both polymers, $4 \mathrm{Bx}$ molecules in their crosslinked state, yielding signals of $-\mathrm{NH}$ component, were found to be distributed uniformly over the film thickness (Fig. 2d and Supplementary Fig. 24a). In addition, both the pristine and crosslinked $\mathrm{P}(\mathrm{DPP} 2 \mathrm{DT}-\mathrm{TVT})$ and $\mathrm{P}(\mathrm{NDI} \mathrm{O} \mathrm{OT}-$ Se2) films showed similar surface morphologies (see the AFM images of these polymer films in Supplementary Fig. 25).

The high crosslinking efficiency of $4 \mathrm{Bx}$ is a key factor allowing only a small loading of the material to produce crosslinked state of the semiconducting polymer films; this is critical for the patterning polymer films while maintaining their optical and electrical characteristics. The photocrosslinking efficiency of $4 \mathrm{Bx}$ crosslinker for $\mathrm{P}(\mathrm{DPP} 2 \mathrm{DT}-\mathrm{TVT})$ and $\mathrm{P}(\mathrm{NDI} 3 \mathrm{OT}-\mathrm{Se} 2)$ was evaluated indirectly by the film retention, defined as the relative change in the optical absorption at wavelength of 817 and 735 $\mathrm{nm}$, respectively, upon rinsing with chloroform where the light absorption is most intense for the respective polymers (Supplementary Fig. 26). If these polymer films are not crosslinked effectively, they would be removed during the rinsing process and thus the film retention would be low. These values are plotted as a function of the weight fraction of $4 \mathrm{Bx}$ added into the film (Fig. 2e). The optical density of the films (that is proportional to the film thickness) prepared from higher contents of $4 \mathrm{Bx}$ could be preserved more effectively, and the polymer films prepared from batches with the highest molecular weight could be preserved using as little as $1 \mathrm{wt} \%$ of the crosslinker (the contents of crosslinker in mol\% relative to the moles of the polymer repeating units are provided as well in Supplementary Table 1), which is the lowest weight fraction reported thus far for crosslinking of polymer semiconductors ${ }^{31,33-36}$. The successful production of a fully crosslinked state using only $1 \mathrm{wt} \%$ of $4 \mathrm{Bx}$ has a significant meaning, because the use of such a small amount of crosslinker would suppress the side effects caused by the photocrosslinking step on the chemical, physical, and electrical properties of semiconducting polymers. Therefore, in the follow-up experiments, $1 \mathrm{wt} \%$ of $4 \mathrm{Bx}$ was utilized, if not noted.

For the structural characterization, two-dimensional grazing incidence X-ray diffraction (2D GIXD) analysis was carried out for photocrosslinked P(DPP2DT-TVT) and P(NDI3OT-Se2) films prepared with different loading of $4 \mathrm{Bx}(0,1,5$, and $10 \mathrm{wt} \%)$. Figure $2 \mathrm{f}$ shows $2 \mathrm{D}$ diffractograms of the series of $\mathrm{P}(\mathrm{DPP} 2 \mathrm{DT}$ TVT) films. The series of P(DPP2DT-TVT) films showed ordered lamellar peaks in the out-of-plane direction and a $\pi-\pi$ stacking peak in the in-plane direction, indicating that $\mathrm{P}(\mathrm{DPP} 2 \mathrm{DT}-\mathrm{TVT})$ is oriented dominantly in an edge-on structure. Figure $2 \mathrm{~g}$ shows the line-cut 1D profiles of the diffractograms along the out-of-plane direction. The peak position observed from the profiles reveals the average spacing between the ordered units in semicrystalline polymer film. The peak intensity allows comparing the crystallinity of semicrystalline films with equivalent scattering volume. The broadness of the peaks (represented by the FWHM) allows estimating the characteristic coherence length $\left(L_{C}\right)$ that scales with the average crystalline domain size of the semicrystalline film. Structural parameters of the films extracted from the diffractograms are summarized in Supplementary Table 2. When high contents of $4 \mathrm{Bx}$ ( 5 and $10 \mathrm{wt} \%$ ) were used, the position of the diffraction peaks did not change compared with that from pristine $\mathrm{P}(\mathrm{DPP} 2 \mathrm{DT}-\mathrm{TVT})$ film, but noticeable reduction in the peak intensity and broadening of the peaks were observed (Fig. $2 \mathrm{~g}, \mathrm{~h}$ ). When P(DPP2DT-TVT) was photocrosslinked using $1 \mathrm{wt} \%$ of $4 \mathrm{Bx}$, on the other hand, the position, intensity, and broadness of the peaks and the associated structural parameters of the films were nearly preserved, compared with those obtained from pristine $\mathrm{P}(\mathrm{DPP} 2 \mathrm{DT}-\mathrm{TVT})$ film with equivalent thickness. This result indicates that only a minute change (if any) in the molecular packing of $\mathrm{P}(\mathrm{DPP} 2 \mathrm{DT}-\mathrm{TVT})$ was induced, when $1 \mathrm{wt} \%$ of $4 \mathrm{Bx}$ was used. Note that this condition of using $1 \mathrm{wt} \%$ of $4 \mathrm{Bx}$ corresponds to the optimal condition that allows photopatterning P(DPP2DT-TVT) with minimal loss in its electrical properties, which will be described below. In sum, these results indicate that the crystallinity of the films is weakened when P(DPP2DT-TVT) is cast together with a large amount of $4 \mathrm{Bx}$ and become crosslinked after exposure to UV. The results also indicate that the inherent crystallinity of the P(DPP2DT-TVT) films can be preserved by using a small amount of $4 \mathrm{Bx}$ due to its high crosslinking efficiency. Despite the structures of the films not being altered noticeably, recall that the crosslinked P(DPP2DTTVT) films using only $1 \mathrm{wt} \%$ of $4 \mathrm{Bx}$ showed excellent retention characteristics against chemical solvents. A consistent behavior was observed for P(NDI3OT-Se2) films (Supplementary Fig. 27).

We further examine the mechanical properties of the photocrosslinked $\mathrm{P}(\mathrm{DPP} 2 \mathrm{DT}-\mathrm{TVT})$ and $\mathrm{P}(\mathrm{NDI}$ OOT-Se2) films by monitoring their strain-stress relation. This was done by employing the film-on-water apparatus on a pseudofreestanding polymer thin films floated on water ${ }^{40,41}$. Figure $2 \mathrm{i}$ shows the stress-strain relations of a series of $\mathrm{P}(\mathrm{DPP} 2 \mathrm{DT}-\mathrm{TVT})$ films 
a

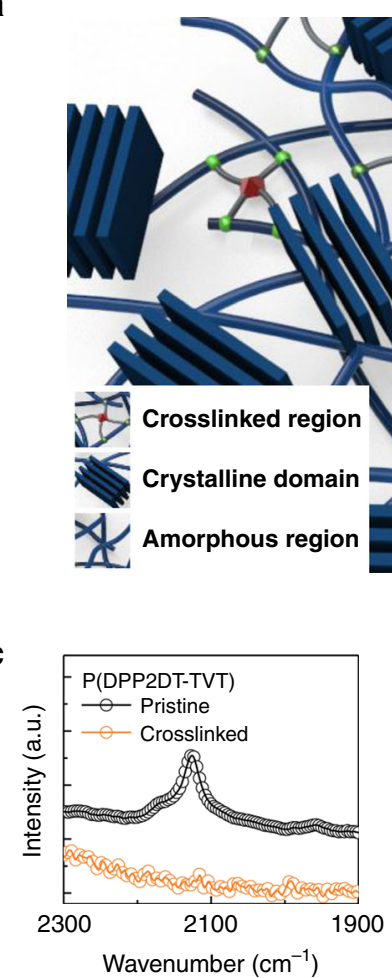

C

d
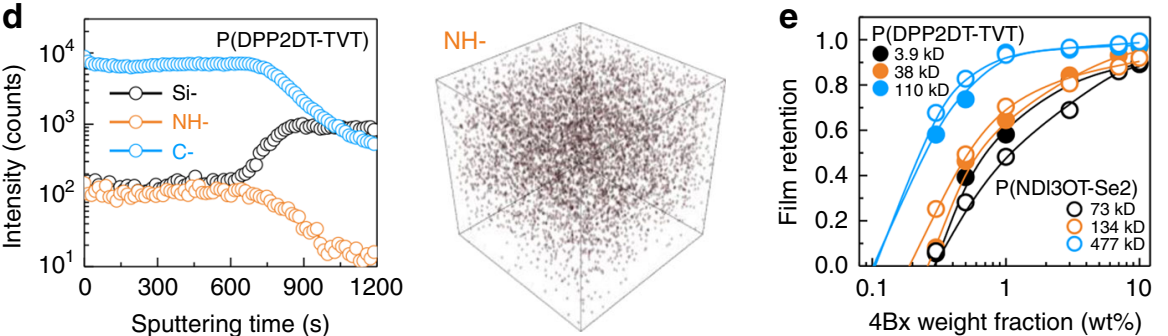

$\mathbf{f}$
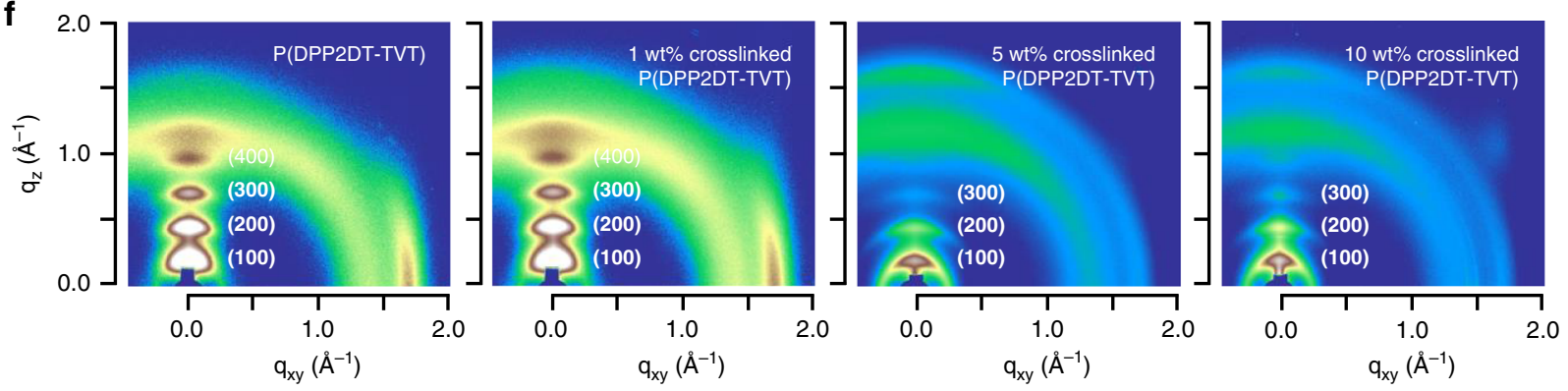

g

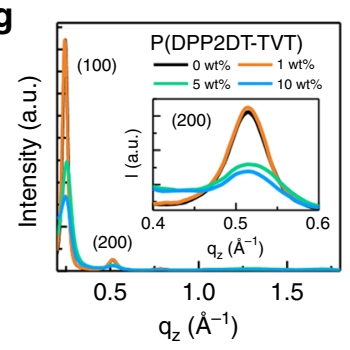

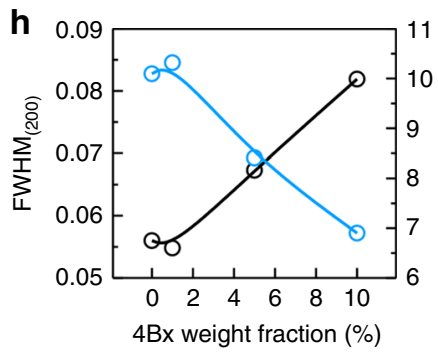

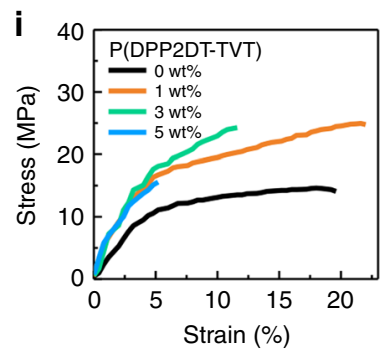

b

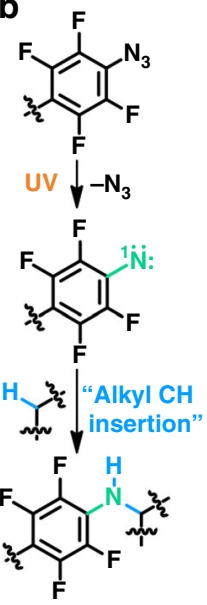

Fig. 2 Structural characterization of crosslinked P(DPP2DT-TVT). a Schematic illustration of a semiconducting polymer in its crosslinked state formed by $4 \mathrm{Bx}$. b The key chemical reaction involved for the crosslinking. c FTIR spectra of P(DPP2DT-TVT) films before and after being crosslinked. $\mathbf{d}$ TOF-SIMS spectra of crosslinked P(DPP2DT-TVT) film monitored as a function of sputtering time. e Film retention characteristics of P(DPP2DT-TVT) and P(NDI3OTSe2) formed with different contents of $4 B x$. $\mathbf{f} 2 D$ GIXD patterns of a series of P(DPP2DT-TVT) films using different loadings of 4Bx. $\mathbf{g}$ The line-cut diffraction profiles of the diffractograms for P(DPP2DT-TVT) films along the of out-of-plane direction. The inset is an enlarged view of the plot near the (200) peak. h Summary of FWHM and characteristic coherence length $\left(L_{C}\right)$ of the out-of-plane (200) peak for P(DPP2DT-TVT) films crosslinked using different loadings of $4 B x$. $\mathbf{i}$ The stress-strain curves of a series of P(DPP2DT-TVT) films crosslinked using different loadings of $4 B x(0,1,3$, and 5 wt $\%$ ). j Tensile modulus of a series of P(DPP2DT-TVT) films crosslinked using different loadings of 4Bx (0, 1, 3, and 5 wt $\%)$.

prepared with different loadings of $4 \mathrm{Bx}(0,1,3$, and $5 \mathrm{wt} \%)$. With increasing the contents of $4 \mathrm{Bx}$, the film became more brittle and its elastic modulus increased, which is another indication that crosslinked polymer structure is developed by using $4 \mathrm{Bx}$ (Fig. 2j). Consistent results were also observed from $\mathrm{P}(\mathrm{NDI} 3 \mathrm{OT}-\mathrm{Se} 2)$ films (Supplementary Fig. 28). In addition, an increase in the glass transition temperature $\left(T_{\mathrm{g}}\right)$ by $8.5^{\circ} \mathrm{C}$ was observed for $\mathrm{P}$ (DPP2DT-TVT) films when they were crosslinked using $5 \mathrm{wt} \%$ of $4 \mathrm{Bx}$ (Supplementary Fig. 29).

The capability to form patterns was then examined upon varying the weight fraction of $4 \mathrm{Bx}$ included in the film; the procedure includes formation of a polymer film containing $4 \mathrm{Bx}$, 
selective exposure of the film to UV through a photomask, and removal of the uncrosslinked region of the film using organic solvent. For comparison, not only the $4 \mathrm{Bx}$ but also the sister crosslinker, ethane-1,2-diyl bis(4-azido-2,3,5,6-tetrafluorobenzoate) $(2 \mathrm{Bx})$ comprising only two crosslinkable units at the termini of the molecules, was evaluated. High-resolution patterns of $\mathrm{P}$ (DPP2DT-TVT) could be achieved using only $1 \mathrm{wt} \%$ of $4 \mathrm{Bx}$ (Supplementary Fig. 30a), thanks to the high crosslinking efficiency of the molecule. By contrast, even the use of $7 \mathrm{wt} \%$ of $2 \mathrm{Bx}$ was not sufficient to form sharp patterns of $\mathrm{P}(\mathrm{DPP} 2 \mathrm{DT}$ TVT) ${ }^{31,33-36}$. Multiple strips of photopatterned P(DPP2DTTVT) films with a spacing of $10 \mu \mathrm{m}$ could also be obtained successfully using $1 \mathrm{wt} \%$ of $4 \mathrm{Bx}$ (Fig. 3a). An AFM image and height profile of the photopatterned P(DPP2DT-TVT) film are shown in Fig. 3b. These films and the images in Fig. 3a, b are obtained from films originally cast by spin-coating method prior to the photopatterning process. We note that the photopatterning process can also be applied to films formed from simple barcoating method that is highly desirable for scale-up. Figure $3 \mathrm{c}$ and Supplementary Fig. 31 show a photograph of a 6 -in. $\mathrm{Si} / \mathrm{SiO}_{2}$ wafer with $\mathrm{P}(\mathrm{DPP} 2 \mathrm{DT}$-TVT) patterns, which were cast onto the substrate by bar-coating process. Figure $3 \mathrm{~d}$ shows the optical microscopy images of the resulting patterns. The results demonstrate that the method is highly adaptable to highthroughput manufacturing.
The electrical properties and stabilities of photocrosslinked semiconducting polymer films were examined using a PTFT device testbed in the bottom-gate top-contact (BGTC) configuration. For the testbed, only the semiconducting polymer layers were photopatterned. Au contacts prepared from thermal evaporation were employed as the source/drain electrodes and an octadecyltrichlorosilane (ODTS)-treated $\mathrm{SiO}_{2} / \mathrm{Si}$ wafer was used as the gate/gate dielectric. Carrier mobility $(\mu)$ of the polymer films was calculated in the saturation regime of the transistor operation according to the equation $I_{\mathrm{D}}=C_{\mathrm{S}} \cdot \mu$. $W \cdot\left(V_{\mathrm{G}}-V_{\mathrm{TH}}\right)^{2} / 2 L^{42-44}$, where $C_{\mathrm{S}}$ is the specific capacitance of the gate dielectric; $V_{\mathrm{TH}}$ is the threshold voltage; and $W$ and $L$ are the channel width and length, respectively. The values of $\mu$ for $\mathrm{P}(\mathrm{DPP} 2 \mathrm{DT}-\mathrm{TVT})$ channels photocrosslinked with $4 \mathrm{Bx}$ and $2 \mathrm{Bx}$ are plotted in Fig. 3e as a function of the crosslinker weight fraction. The hole mobilities decreased with increasing the weight fraction of crosslinkers. The poorer crystallinity of the film as well as the insertion of a larger amount of insulating crosslinker molecule would interrupt charge transport in the photocrosslinked films ${ }^{45}$. It is noteworthy that P(DPP2DT-TVT) films that are crosslinked only with $1 \mathrm{wt} \%$ of $4 \mathrm{Bx}$ showed nearly the same hole mobility as those of pristine P(DPP2DT-TVT) films. This can be well explained with the fact that photocrosslinking with the use of $1 \mathrm{wt} \% 4 \mathrm{Bx}$ did not make any morphological changes in the film structure as explained above. Actually, the hole mobility
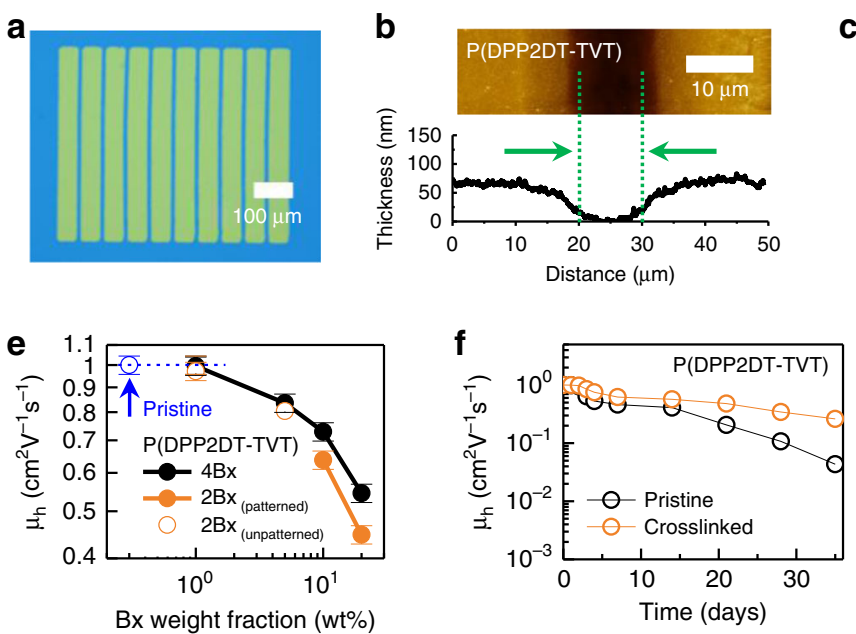

C

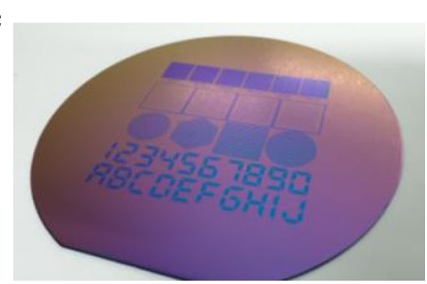

d

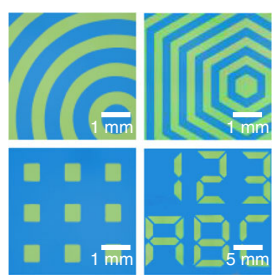

g
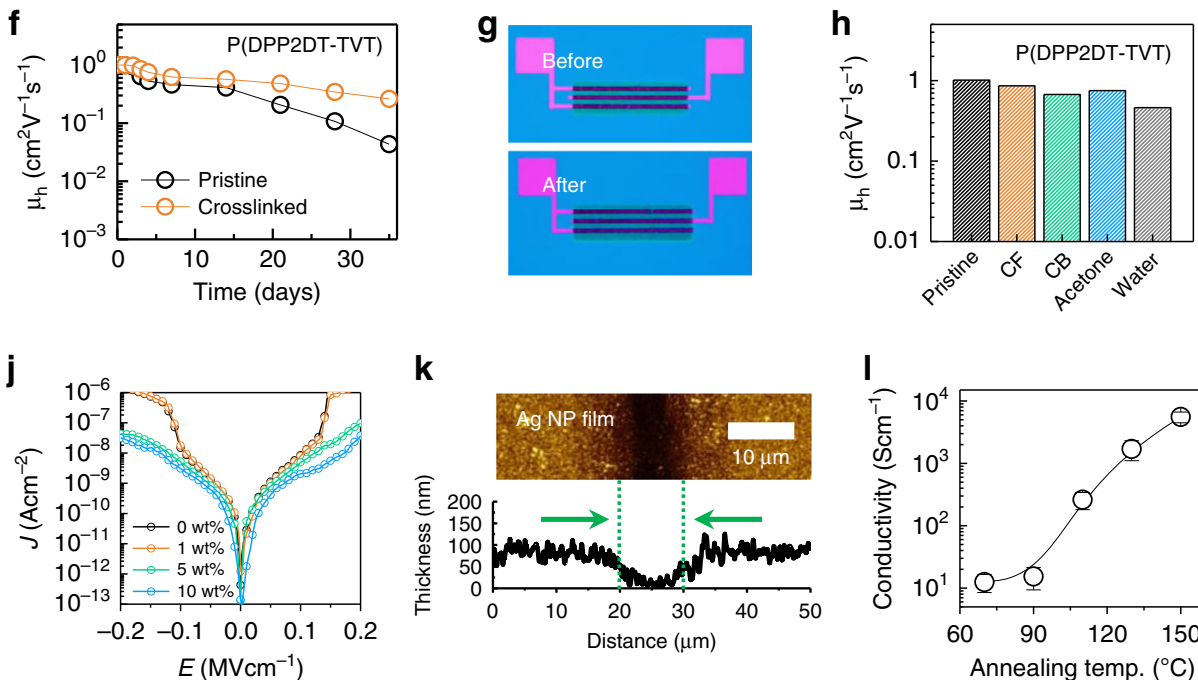

$\mathbf{k}$

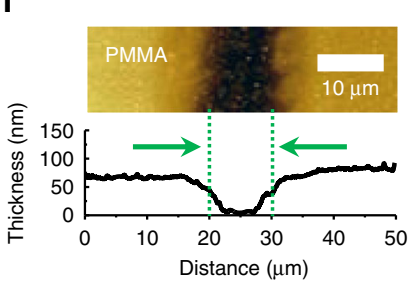

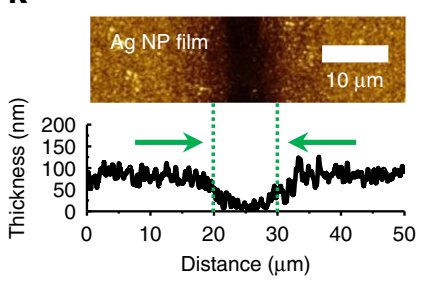

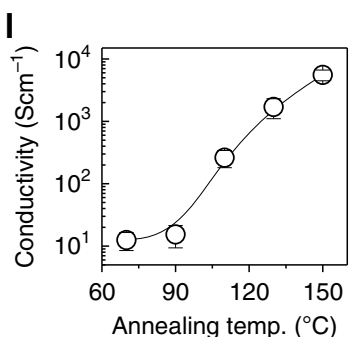

Fig. 3 Characterization of electronic components photopatterned using 4Bx. a OM image of photopatterned P(DPP2DT-TVT) films with a spacing of 10 $\mu \mathrm{m}$. b AFM image and height profile of photopatterned P(DPP2DT-TVT) films. c Photograph of a 6 -in. $\mathrm{Si}_{/} \mathrm{SiO}_{2}$ wafer with P(DPP2DT-TVT) patterns. The polymer film was cast onto the wafer by bar-coating process before the photopatterning process. $\mathbf{d}$ Optical microscopy top-view image of the patterns of P(DPP2DT-TVT) shown in c. e Hole mobilities for PTFTs based on photocrosslinked P(DPP2DT-TVT) channel plotted as a function of the loading of the two crosslinkers ( $4 \mathrm{Bx}$ and $2 \mathrm{Bx}$ ). Note that the data in open circle are obtained from a photocrosslinked P(DPP2DT-TVT) channel prepared using 1 wt $\%$ of $2 \mathrm{Bx}$. The resulting films did not exhibit good retention characteristics, and thus the films prepared using $1 \mathrm{wt} \%$ of $2 \mathrm{Bx}$ cannot be utilized for the patterning process. $\mathbf{f}$ Comparison of the hole mobilities over time for pristine and crosslinked P(DPP2DT-TVT) PTFTs. $\mathbf{g}$ OM images of a PTFT based on photopatterned P(DPP2DT-TVT) before and after being soaked in CF. $\mathbf{h}$ Hole mobilities of PTFTs based on photopatterned P(DPP2DT-TVT) before and after being soaked in various solvents. i AFM image and height profile of photopatterned PMMA film. $\mathbf{j}$ Current density-electric field plot of PMMA films crosslinked using different loadings of $4 \mathrm{Bx}$. $\mathbf{k}$ AFM image and height profile of photopatterned Ag NP film. I Electrical conductivity of Ag NP films annealed at different temperatures. 
of P(DPP2DT-TVT) films crosslinked with $1 \mathrm{wt} \%$ of $2 \mathrm{Bx}$ showed a similar value. However, unlike $4 \mathrm{Bx}$, using only $1 \mathrm{wt} \%$ of $2 \mathrm{Bx}$ did not form $\mathrm{P}(\mathrm{DPP} 2 \mathrm{DT}-\mathrm{TVT})$ films with good film retention characteristics against chloroform, which is mandatory for the photopatterning process. Patterning is only possible when crosslinking of polymers is done sufficiently so that the resulting film gains good retention characteristics against subsequent developing processes (Supplementary Fig. 30a). For 2Bx, $>7 \mathrm{wt}$ $\%$ was required to form chemically robust $\mathrm{P}$ (DPP2DT-TVT) films, which, in turn, lead to the severe reduction in hole mobility. A similar trend was also observed for $n$-type PTFTs based on the $\mathrm{P}(\mathrm{NDI} 3 \mathrm{OT}-\mathrm{Se} 2$ ) film photopatterned by $4 \mathrm{Bx}$ (Supplementary Fig. 32). These observations altogether highlight that the use of $4 \mathrm{Bx}$ does not lead to suppression in the electrical properties of the host semiconducting polymers even after photopatterning.

In addition, the stabilities of the pristine and crosslinked PTFTs were compared. First, the shelf life stability of the PTFTs was examined by monitoring the mobility of the devices over time. It was observed that the initial hole mobility was better preserved for the crosslinked films than for the pristine films (Fig. 3f and Supplementary Fig. 33). The higher shelf life stability of PTFTs based on crosslinked polymer channel is perhaps due to the slower diffusion of oxygen or water molecules within the photocrosslinked polymer film. Second, the chemical stability of the PTFTs was examined. This was done by comparing the performances of photopatterned PTFTs based on P(DPP2DTTVT) and P(NDI3OT-Se2) channels crosslinked with $1 \mathrm{wt} \%$ of $4 \mathrm{Bx}$ with those obtained after soaking the devices into four different solvents (CF, chlorobenzene, acetone, and deionized water) for $5 \mathrm{~min}$. No apparent change was observed from optical microscopy images for the photocrosslinked P(DPP2DT-TVT) PTFTs (Fig. 3g), and their carrier mobilities were only affected slightly (Fig. 3h). Similar results were obtained after solventsoaking the photocrosslinked P(NDI3OT-Se2) PTFTs (Supplementary Fig. 34). In contrast, the carrier mobilities of the PTFTs based on uncrosslinked, pristine polymer channels decreased dramatically after solvent-soaking (Supplementary Fig. 35). These results indicate that electrical properties of the photocrosslinked polymer channels can be preserved during the developing process, i.e., the step of removing the uncrosslinked regions of the polymer thin films using chemical solvents during the patterning process.

$4 \mathrm{Bx}$ was also used to photopattern insulating polymers, such as PMMA, PS, and PVDF-HFP (Supplementary Fig. 30b), which can serve as the gate dielectric layer of PTFTs. Consistent with the crosslinking done for the semiconducting polymers, these insulating polymers could be photocrosslinked according to the $\mathrm{C}-\mathrm{H}$ insertion reaction of the $\mathrm{UV}$-activated nitrene groups of $4 \mathrm{Bx}$ uniformly across the entire thickness of the film (Supplementary Figs. 23b, c and 24b). The AFM image and height profile of a photopatterned PMMA film are shown in Fig. 3i, which demonstrates that patterns with a feature size $<10 \mu \mathrm{m}$ can be formed using $4 \mathrm{Bx}$. The frequency-dependent capacitance, representing the dielectric properties of an insulator, was identical for the pristine and crosslinked PMMA films (Supplementary Fig. 36). This indicates that the photopatterning process did not cause a series adverse effect on the capacitive properties of the material. The current density $(J)$-electric field $(E)$ curves for the PMMA films (thickness $=480 \mathrm{~nm}$ ) in a metal/insulator $/$ metal (MIM) device geometry were also obtained, which were incorporated with various amounts of the $4 \mathrm{Bx}(0-10 \mathrm{wt} \%)$. Using a small amount of $4 \mathrm{Bx}(<5 \mathrm{wt} \%)$ resulted in a large leakage current for the crosslinked PMMA films at an electric field of $>0.1 \mathrm{MV} \mathrm{cm}^{-1}$ (Fig. 3j). The leakage current was significantly suppressed when $>5 \mathrm{wt} \%$ of $4 \mathrm{Bx}$ was used. This result suggests that the leakage current through the PMMA films can be effectively reduced upon the formation of a tight structural network via a high degree of crosslinking. Finally, $4 \mathrm{Bx}$ could be used to photopattern Ag NPs. The Ag NPs are composed of core Ag atoms surrounded with aliphatic surfactants (Supplementary Fig. 37), and the aliphatic surfactants can undergo the crosslinking reaction with the use of $4 \mathrm{Bx}$ under UV. Films of Ag NPs could thus be photopatterned by using $5 \mathrm{wt} \%$ of $4 \mathrm{Bx}$ with a narrow feature size $(\sim 10 \mu \mathrm{m})$ (Fig. $3 \mathrm{k})$. Once the Ag NP films were patterned, they were annealed at different temperatures $\left(70-150^{\circ} \mathrm{C}\right)$. Conductivity as high as $5500 \mathrm{~S} \mathrm{~cm}^{-1}$ was obtained from $\mathrm{Ag}$ NP films annealed at $150^{\circ} \mathrm{C}$ (Fig. 3l). This conductivity is high enough for the Ag NP films to be used as the electrodes for PTFTs, while the annealing temperature is low enough for the films to be processed onto a flexible plastic substrate. Overall, we demonstrated that $4 \mathrm{Bx}$ can be added to the entire electronic components of PTFTs, i.e., $n$ - or $p$-type channel layer, gate dielectric layer, and electrodes, allowing photopatterning each of these component layers.

Electrical characteristics of all-photopatterned PTFTs and logic circuits. Exploiting the optimized processes developed for each of these components, all-photopatterning of PFTFs and logic circuits based on these devices was conducted, according to the protocol described in Fig. 1c. The output characteristics [drain current $\left(I_{\mathrm{D}}\right)$ versus drain voltage $\left.\left(V_{\mathrm{D}}\right)\right]$ of a single allphotopatterned $p$-type PTFT based on P(DPP2DT-TVT) channel, PMMA gate dielectric (specific capacitance $=5 \mathrm{nF} \mathrm{cm}^{-2}$ ), and Ag NP electrodes at six different gate voltages $\left(V_{\mathrm{G}} \mathrm{s}\right)$ are shown in Fig. 4a. The curves demonstrate the gate modulation with a linear behavior at low- $V_{\mathrm{D}}$ region and a saturation behavior at high- $V_{\mathrm{D}}$ region. The observation of the linear behavior at low$V_{\mathrm{D}}$ region indicates that an Ohmic contact is formed between the P(DPP2DT-TVT) channel and Ag NP electrodes. The transfer characteristics $\left(I_{\mathrm{D}}\right.$ versus $\left.V_{\mathrm{G}}\right)$ of the $p$-type PTFT at a fixed $V_{\mathrm{D}}$ of $-60 \mathrm{~V}$ are shown in Fig. $4 \mathrm{~b} .\left|I_{\mathrm{D}}\right|$ increased with increasing $V_{\mathrm{G}}$ negatively, which is a typical signature of $p$-type transistor operation. No obvious hysteresis was observed between the forward and reverse sweeps, implying a small density of trap sites presenting at the semiconductor/dielectric interface. The electrical properties of 36 all-photopatterned P(DPP2DT-TVT) PTFTs fabricated in a single batch of experiment are summarized in Fig. 4c. The average hole mobility was estimated to be 0.81 $( \pm 0.18) \mathrm{cm}^{2} \mathrm{~V}^{-1} \mathrm{~s}^{-1}$. It is noteworthy that a maximum mobility of $1.03 \mathrm{~cm}^{2} \mathrm{~V}^{-1} \mathrm{~s}^{-1}$ was obtained even though all electronic components were photopatterned. Average on-off current ratio was $1.6( \pm 0.8) \times 10^{6}$. Average $V_{\mathrm{TH}}$ was $-56( \pm 4) \mathrm{V}$. Average turnon voltage $\left(V_{\text {on }}\right)$, defined as the gate voltage yielding the onset of the channel current was $+7.7( \pm 0.5) \mathrm{V}$. Nearly equivalent device performance was obtained from all-photopatterned P(DPP2DTTVT) TFTs using the same materials but in the BGTC configuration (Supplementary Fig. 38). Furthermore, a polymer gate dielectric with higher capacitance, e.g., a photopatterned PVDF-HFP layer (specific capacitance $=11.1 \mathrm{nF} \mathrm{cm}^{-2}$ ), could be employed for the device, demonstrating the reduction of the operation voltages. The average $V_{\mathrm{TH}}$ and $V_{\text {on }}$ for the allphotopatterned TFTs with PVDF-HFP gate dielectric were reduced to -11 and $+0.7 \mathrm{~V}$, respectively, and these devices could operate at reduced gate voltages below $20 \mathrm{~V}$ (Supplementary Fig. 39).

The $n$-type PTFTs were fabricated using P(NDI3OT-Se2), PMMA gate dielectric, and Ag NP electrodes. The output characteristics of a single $\mathrm{P}(\mathrm{NDI} 3 \mathrm{OT}-\mathrm{Se} 2)$ TFT show an increase in $I_{\mathrm{D}}$ with a positive increase in $V_{\mathrm{G}}$, which is a signature of $n$-type transistor operation (Fig. 4d). The representative transfer characteristic of the $\mathrm{P}(\mathrm{NDI} 3 \mathrm{OT}-\mathrm{Se} 2)$ TFT at $V_{\mathrm{D}}=+60 \mathrm{~V}$ is 

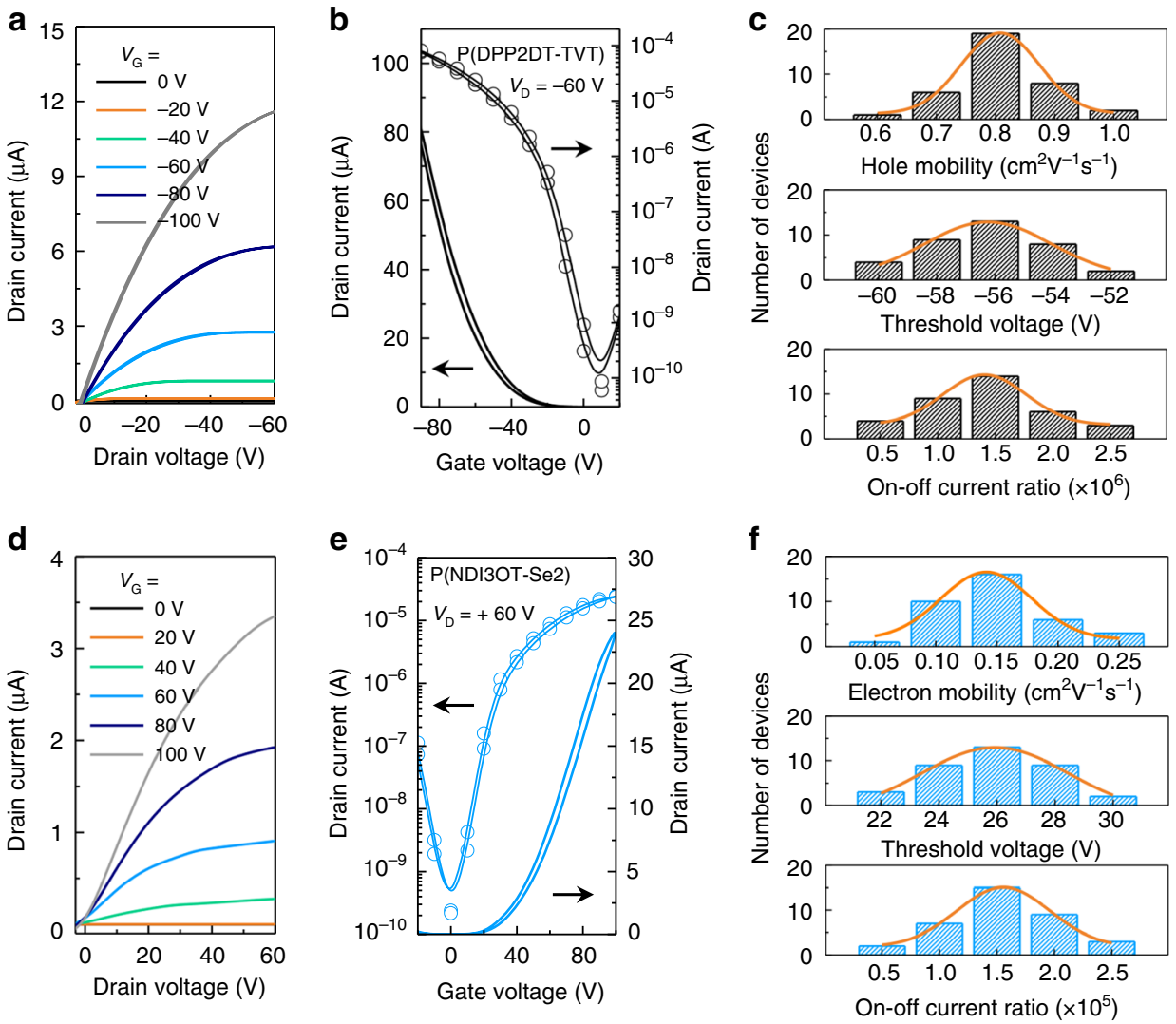

Fig. 4 Electrical characteristics of all-photopatterned PTFTs. a Output and $\mathbf{b}$ transfer characteristics of PTFTs based on P(DPP2DT-TVT) crosslinked using $1 \mathrm{wt} \%$ of $4 \mathrm{Bx}$. c Summary of hole mobility (top), threshold voltage (middle), and on-off current ratio (bottom) of 36 P(DPP2DT-TVT) PTFTs. d Output and $\mathbf{e}$ transfer characteristics of PTFTs based on P(NDI3OT-Se2) crosslinked using 1 wt $\%$ of 4 Bx. f Summary of electron mobility (top), threshold voltage (middle), and on-off current ratio (bottom) of 36 P(NDI3OT-Se2) PTFTs.

Table 1 Summary of electrical characteristics of 36 all-solution-processed, all-photopatterned PTFTs.

\begin{tabular}{|c|c|c|c|}
\hline Semiconducting polymer & Carrier mobility $\left(\mathrm{cm}^{2} \mathrm{v}^{-1} \mathrm{~s}^{-1}\right)$ & On-off current ratio & Threshold voltage \\
\hline P(DPP2DT-TVT) & $0.81( \pm 0.18) \mathbf{1 . 0 3}$ & $1.6( \pm 0.8) \times 10^{6}$ & $-56( \pm 4) \vee$ \\
\hline P(DPP2DT-T2) & $0.25( \pm 0.09) \mathbf{0 . 3 5}$ & $1.4( \pm 1.1) \times 10^{6}$ & $-60( \pm 3) V$ \\
\hline P(NDI3OT-Se2) & $0.15( \pm 0.09) \mathbf{0 . 2 4}$ & $1.5( \pm 0.9) \times 10^{5}$ & $26( \pm 4) \vee$ \\
\hline P(NDI2OD-F2T2) & $0.10( \pm 0.03) \mathbf{0 . 1 3}$ & $2.1( \pm 1.1) \times 10^{6}$ & $47( \pm 7) \vee$ \\
\hline $\mathrm{P}(\mathrm{ND} \mid 2 \mathrm{OD}-\mathrm{Se} 2)$ & $0.14( \pm 0.05) 0.19$ & $6.1( \pm 0.4) \times 10^{5}$ & $74( \pm 8) \vee$ \\
\hline
\end{tabular}

shown in Fig. 4e. The histograms obtained from 36 allphotopatterned $n$-type PTFTs reveal average electron mobility of $0.15( \pm 0.09) \mathrm{cm}^{2} \mathrm{~V}^{-1} \mathrm{~s}^{-1}, V_{\mathrm{TH}}$ of $26( \pm 4) \mathrm{V}, V_{\mathrm{on}}$ of -0.6 $( \pm 0.4) \mathrm{V}$, and on-off current ratio of $1.5( \pm 0.9) \times 10^{5}$. Consistent with the $p$-type devices, the all-photopatterned $n$-type PTFTs employing a high capacitance PVDF-HFP gate dielectric layer operated at reduced gate voltages below $20 \mathrm{~V}$ (Supplementary Fig. 39 and Supplementary Table 3). PTFTs based on six other semiconducting polymers were fabricated by the same method using $1 \mathrm{wt} \%$ of $4 \mathrm{Bx}$ (Supplementary Fig. 40), and their device characteristics are summarized in Table 1. These data demonstrate that our photopatterning approach using $4 \mathrm{Bx}$ is generally applicable to a variety of polymer semiconductors.

Fabrication of all-photopatterned $p$-type and $n$-type PTFTs with reliable operation enabled assembling these devices into functional complementary logic circuits, as shown in Fig. $5^{46,47}$.
OM images and the corresponding circuit diagrams of these allphotopatterned complementary logic circuits, i.e., NOT, NAND, and NOR logic gates, are shown in Fig. 5a. First, the NOT gate was fabricated by connecting a $p$-type and an $n$-type PTFTs in series. The voltage transfer characteristics of the NOT gate at various supply voltages $\left(V_{\mathrm{DD}} \mathrm{s}\right)$ of $10,20,30,40,50$, and $60 \mathrm{~V}$ are shown in Fig. 5b. When the input voltage $\left(V_{\mathrm{IN}}\right)$ was in the logic state " 0 " $\left(V_{\mathrm{IN}}=0 \mathrm{~V}\right)$, the output voltage $\left(V_{\text {OUT }}\right)$ yielded the logic state " 1 " $\left(V_{\text {OUT }}=V_{\mathrm{DD}}\right)$. Signal inversion was clearly observed with a positive increase in $V_{\text {IN }}$ such that $V_{\text {OUT }}$ decreased to $0 \mathrm{~V}$ (logic state "0"). The signal inverter gain of the NOT gate, defined as $\left|d V_{\mathrm{OUT}} / d V_{\mathrm{IN}}\right|$, was determined to be $\sim 10$ at $V_{\mathrm{DD}}=60 \mathrm{~V}$. The output signal obtained at $V_{\mathrm{DD}}=60 \mathrm{~V}$ is shown in Fig. $5 \mathrm{c}$. $V_{\text {OUT }}$ switched from the " 1 " state to the " 0 " state when $V_{\text {IN }}$ was switched from the " 0 " state to the " 1 " state, and vice versa. More complicated logic circuits, such as NAND and NOR gates, could 
a
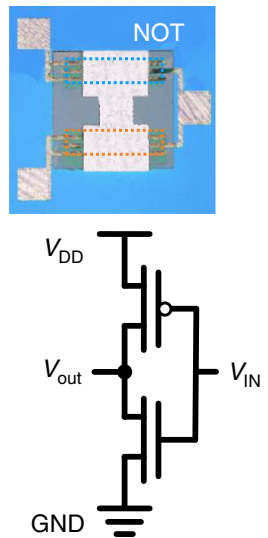

b
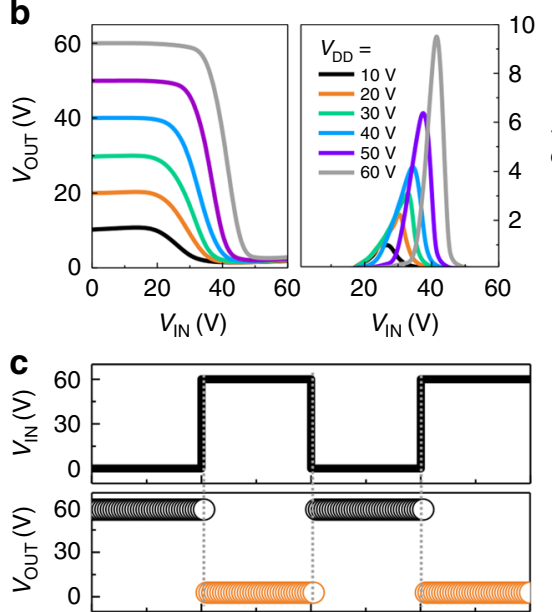

--- P(DPP2DT-TVT) - --- P(NDI3OT-Se2)
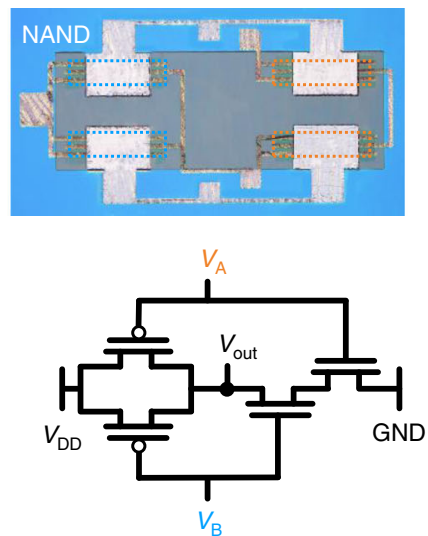
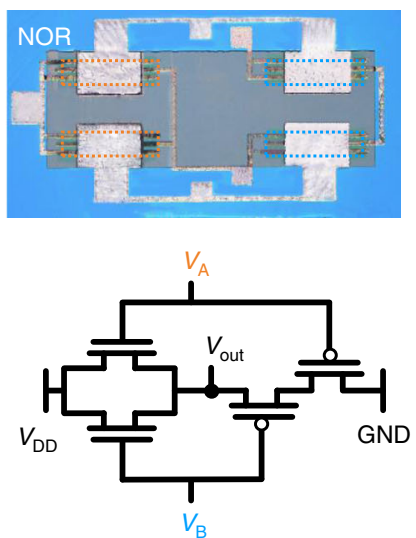

d

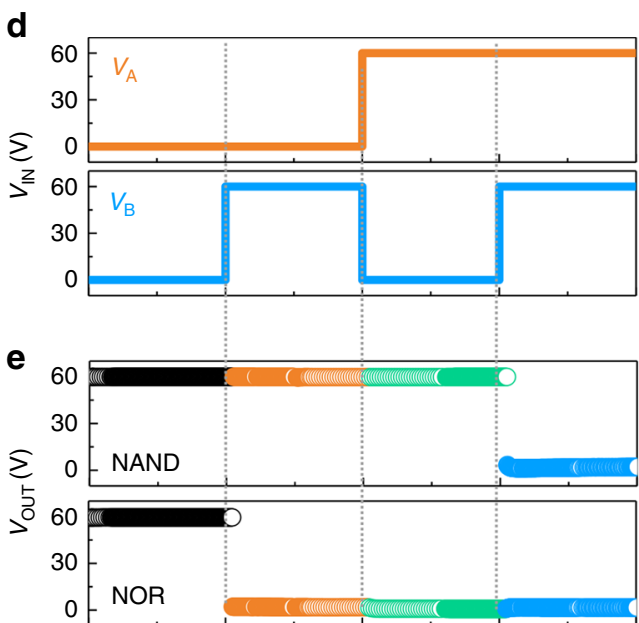

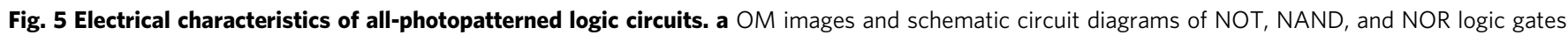

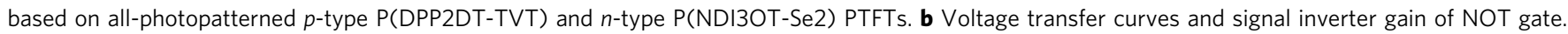

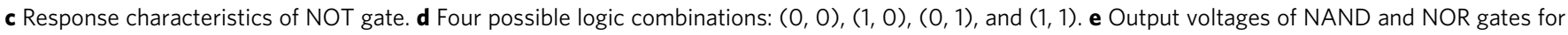
the four logic combinations in $\mathbf{d}$.

also be fabricated by assembling four PTFTs. The NAND gate was fabricated by connecting two $n$-type PTFTs in series and two $p$-type PTFTs in parallel, whereas the NOR gate was fabricated by connecting two $n$-type PTFTs in parallel and two $p$-type PTFTs in series. The four possible input logic combinations, $(0,0),(1,0)$, $(0,1)$, and $(1,1)$, are shown in Fig. $5 d$; here, the input voltages $V_{\mathrm{A}}$ and $V_{\mathrm{B}}$ were switched between 0 and $60 \mathrm{~V}$ at a constant $V_{\mathrm{DD}}$ of $+60 \mathrm{~V}$. As observed in Fig. 5e, both the NAND gate and the NOR gate showed different output signals at different combinations of input signals. For the NAND gate, $V_{\text {OUT }}$ was in the logic state "0" only for the $(1,1)$ logic combination, and it was in the logic state " 1 " for the other three logic combinations, i.e., $(0,0),(1,0)$, and $(0,1)$. For the NOR gate, on the other hand, $V_{\text {OUT }}$ was in the logic state " 1 " only for the $(0,0)$ logic combination, and it was in the logic state " 0 " for the other three logic combinations, i.e., $(1,0)$, $(0,1)$, and $(1,1)$. Both these complementary logic gates showed ideal rail-to-rail output voltages in the corresponding input states. These results demonstrate the logic circuits prepared from allsolution, all-photopatterned process which should be expendable to even a complicated circuitry design.

\section{Discussion}

We herein described a photopatterning approach based on $4 \mathrm{Bx}$ with high crosslinking efficiency for realizing all-solutionprocessed organic electronic devices. Different types of electronic materials, i.e., semiconducting polymers, insulating polymers, and metal NPs, were successfully photocrosslinked using a very small amount of $4 \mathrm{Bx}$. Semiconducting polymers could be successfully photocrosslinked using only $1 \mathrm{wt} \%$ of $4 \mathrm{Bx}$ without altering their intrinsic crystallinity and charge transport properties. Moreover, the good solvent resistance of the photocrosslinked films resulted in high-resolution polymer micropatterns. This pivotal feature facilitated multistacking of the photopatterned electronic component layers and the fabrication of an array of all-solutionprocessed, all-photopatterned PTFT devices. NOT, NAND, and NOR logic gates could be successfully fabricated by allphotopatterning $p$-type and $n$-type PTFTs. Overall, this work demonstrates an effective route to all-solution-processed organic electronic devices based on a single fabrication protocol.

\section{Methods}

Film characterizations. The photochemical reaction of $4 \mathrm{Bx}$ in the electronic component layers was confirmed by FTIR spectroscopy (Bruker IFS-66/S, TENSOR27). The spatial distribution of the crosslinking points was identified by TOFSIMS (ION-TOF, Germany). $\mathrm{Bi}_{3}{ }^{2+}(30 \mathrm{keV})$ was used as the primary ion source in TOF-SIMS. Negative secondary ions from an area of $100 \times 100 \mu \mathrm{m}^{2}$ were detected. Sputter etching was performed over an area of $400 \times 400 \mu \mathrm{m}^{2}$ using an Ar gas cluster ion beam with an acceleration voltage of $2.5 \mathrm{keV}$. The currents of the primary ion beam and Ar gas cluster ion beam were $0.5 \mathrm{pA}$ and $0.9 \mathrm{nA}$, respectively, for the polymer films and $0.9 \mathrm{pA}$ and $40.0 \mathrm{nA}$, respectively, for the Ag NP film. The crystalline structures of the semiconducting polymer films were examined by 2D GIXD at the 9A beamline of the Pohang Light Source II, Republic of Korea. Tapping-mode AFM images were obtained using Multimode-N3-AM (Bruker/ 
Veeco/Digital Instruments). UV-visible absorption spectra were recorded on the UV-3600 spectrophotometer (Shimadzu). The glass transition temperature was measured by spectroscopic ellipsometry (SEMG-1000, Nano-view) with a thermal stage $\left(2{ }^{\circ} \mathrm{C}\right.$ per min) under vacuum. The electrical properties of the PTFTs were measured at room temperature under dark using a Keithley 4200 semiconductor characterization system. Specific capacitances of the polymer gate dielectrics were measured in the MIM device geometry using the Agilent E-4980a LCR meter in the frequency range of $100 \mathrm{~Hz}$ to $1 \mathrm{MHz}$. For the fabrication of capacitors, PMMA was deposited onto a heavily doped $\mathrm{Si}$ substrate, and this was followed by the thermal evaporation of top Au electrodes $(\sim 40 \mathrm{~nm})$. The area of each Au electrode was $500 \mu \mathrm{m}^{2}$. Crosslinked $\mathrm{Ag} \mathrm{NP}$ film was sintered at different temperatures $\left(70-150^{\circ} \mathrm{C}\right)$ in a vacuum oven $\left(\sim 10^{-3}\right.$ Torr $)$ for $8 \mathrm{~h}$. The electrical conductivity of the sintered $\mathrm{Ag}$ NP film was measured by the four-point probe method (Keithley 2182A and 6221).

Fabrication of BGTC PTFT testbed devices. For testing of the electrical properties and stabilities of photocrosslinked polymers, BGTC PTFTs were fabricated on a heavily $n$-doped $\mathrm{Si}$ wafer having a thermally grown 300 -nm-thick $\mathrm{SiO}_{2}$ layer. The $\mathrm{SiO}_{2} / \mathrm{Si}$ substrate was cleaned by ultrasonication with acetone, isopropanol, and deionized water in sequence for $10 \mathrm{~min}$ each. The $\mathrm{SiO}_{2}$ surface was treated with ODTS (Gelest, Inc.) to prevent charge trapping by silanol groups on the $\mathrm{SiO}_{2}$ surface. Blend solutions of $p$-type P(DPP2DT-TVT) or $n$-type P(NDI3OT-Se2) polymer and various amounts of crosslinker $(4 \mathrm{Bx}$ or $2 \mathrm{Bx})$ with a concentration of $5 \mathrm{mg} \mathrm{mL}^{-1}$ were spin-coated onto the ODTS-treated $\mathrm{SiO}_{2} / \mathrm{Si}$ substrate. After photopatterning $\left(254 \mathrm{~nm}, 1000 \mathrm{~W} \mathrm{~cm}^{-2}, 30 \mathrm{~s}\right)$ using a shadow mask inside a $\mathrm{N}_{2}$ filled glove box, the photopatterned polymer films were stored for $4 \mathrm{~h}$ to remove the residual solvent. Finally, 50-nm-thick Au S/D electrodes were thermally deposited onto the photocrosslinked semiconducting polymer films through a shadow mask. The channel length $(L)$ and width $(W)$ of the S/D electrodes were 100 and $800 \mu \mathrm{m}$, respectively.

\section{Fabrication of TGBC all-photopatterned PTFTs and logic gate array. PEN} (Teijin DuPont Films, Teonex Q65HA) was used as the plastic substrate after cleaning by ultrasonication with acetone, isopropanol, and deionized water in sequence for 10 min each. Ag NP dispersion (Nanopaste, NPS-J, Harima Chemicals Group, Inc., Japan) was dried, and then redissolved in anhydrous CF (Sigma Aldrich, $\geq 99 \%$ ) at a concentration of $50 \mathrm{mg} \mathrm{mL}^{-1}$. Then, $5 \mathrm{wt} \%$ of $4 \mathrm{Bx}$ was added to the Ag NP solution in CF. To form the Ag NP S/D electrodes, a mixture solution of $\mathrm{Ag}$ NPs and $4 \mathrm{Bx}$ was spin-coated onto the PEN substrate at $1000 \mathrm{rpm}$ for $30 \mathrm{~s}$ inside a $\mathrm{N}_{2}$-filled glove box. The resulting Ag NP film was exposed to a UV lamp (wavelength: $254 \mathrm{~nm}$, power: $1000 \mathrm{~W} \mathrm{~cm}^{-2}$ ) for $30 \mathrm{~s}$ through a shadow mask. After photocrosslinking, the uncrosslinked regions were removed by spin-coating a fresh $\mathrm{CF}$ onto the film at $4000 \mathrm{rpm}$. As a result, the photocrosslinked regions of the $\mathrm{Ag}$ NP films were patterned directly on the PEN substrate and then thermally sintered in a vacuum oven at $150^{\circ} \mathrm{C}$ for $8 \mathrm{~h}$. The thickness of the sintered $\mathrm{Ag} \mathrm{NP}$ electrode was around $72 \mathrm{~nm}$. The channel length $(L)$ and width $(W)$ of the S/D electrodes were 100 and $800 \mu \mathrm{m}$, respectively. The photopatterning of all the subsequent components was performed in the same manner. In the case of the semiconducting polymer solutions, complete crosslinking could be successfully achieved by the addition of only $1 \mathrm{wt} \%$ of $4 \mathrm{Bx}$. A $5 \mathrm{mg} \mathrm{mL}^{-1}$ blend solution of the $p$-type $\mathrm{P}(\mathrm{DPP} 2 \mathrm{DT}-\mathrm{TVT})$ polymer and $4 \mathrm{Bx}(1 \mathrm{wt} \%)$ was spin-coated onto the PEN substrate with the photopatterned Ag NP S/D electrodes. After the photopatterning of the $p$-type polymer film, the $n$-type $\mathrm{P}(\mathrm{NDI} 3 \mathrm{OT}$-Se2) polymer film was formed by spin-coating a $5 \mathrm{mg} \mathrm{mL}^{-1}$ blend solution of $n$-type P(NDI3OT-Se2) and $4 \mathrm{Bx}(1 \mathrm{wt}$ $\%)$. After the photopatterning of the $n$-type polymer film, both the photopatterned semiconducting polymer films were stored inside a glove box for $4 \mathrm{~h}$ to remove residual solvent. A $70 \mathrm{mg} \mathrm{mL}^{-1}$ mixed solution (PMMA (Sigma Aldrich, 120 $\mathrm{kDa}): 4 \mathrm{Bx}=95: 5 \mathrm{wt} \%$ ) in $n$-butyl acetate (Sigma Aldrich) was spin-coated to form the gate dielectric layer. After the photopatterning process of this layer, it was dried at $80^{\circ} \mathrm{C}$ for $6 \mathrm{~h}$ in a vacuum oven. The thickness of the PMMA layer was around $482 \mathrm{~nm}$. Finally, Ag NP gate electrodes were formed by the same method that was applied to form the Ag NP S/D electrodes.

\section{Data availability}

All data generated or analyzed during this study are included in this published article (and its Supplementary Information files).

Received: 18 September 2019; Accepted: 17 February 2020;

Published online: 23 March 2020

\section{References}

1. Gelinck, G. H. et al. Flexible active-matrix displays and shift registers based on solution-processed organic transistors. Nat. Mater. 3, 106-110 (2004).

2. Oh, J. Y. et al. Intrinsically stretchable and healable semiconducting polymer for organic transistors. Nature 539, 411-415 (2016).
3. Yan, H. et al. A high-mobility electron-transporting polymer for printed transistors. Nature 457, 679-686 (2009).

4. Sirringhaus, H. 25th anniversary article: organic field-effect transistors: the path beyond amorphous silicon. Adv. Mater. 26, 1319-1335 (2014).

5. Kettner, M. et al. Solution-processed organic transistors with excellent electrical stability under ambient conditions. Adv. Electron. Mater. 5, 1900295 (2019).

6. Wang, S. et al. Skin electronics from scalable fabrication of an intrinsically stretchable transistor array. Nature 555, 83-88 (2018).

7. Yao, Y., Zhang, L., Leydecker, T. \& Samorì, P. Direct photolithography on molecular crystals for high performance organic optoelectronic devices. J. Am. Chem. Soc. 140, 6984-6990 (2018).

8. Rogers, J. A., Someya, T. \& Huang, Y. Materials and mechanics for stretchable electronics. Science 327, 1603-1607 (2010).

9. $\mathrm{Xu}, \mathrm{X}$. et al. Electron mobility exceeding $10 \mathrm{~cm}^{2} \mathrm{~V}^{-1} \mathrm{~s}^{-1}$ and band-like charge transport in solution-processed $\mathrm{n}$-channel organic thin-film transistors. Adv. Mater. 28, 5276-5283 (2016).

10. Zhang, G. et al. Fused heptacyclic-based acceptor-donor-acceptor small molecules: n-substitution toward high-performance solution-processable fieldeffect transistors. Chem. Mater. 31, 2027-2035 (2019).

11. Paterson, A. F. et al. Small molecule/polymer blend organic transistors with hole mobility exceeding $13 \mathrm{~cm}^{2} \mathrm{~V}^{-1} \mathrm{~s}^{-1}$. Adv. Mater. 28, 7791-7798 (2016).

12. Thomas, T. H. et al. Short contacts between chains enhancing luminescence quantum yields and carrier mobilities in conjugated copolymers. Nat. Commun. 10, 2614 (2019).

13. Nikolka, M. et al. High-mobility, trap-free charge transport in conjugated polymer diodes. Nat. Commun. 10, 2122 (2019).

14. Fahlman, M. et al. Interfaces in organic electronics. Nat. Rev. Mater. 4, 627-650 (2019).

15. Khodagholy, D. et al. High transconductance organic electrochemical transistors. Nat. Commun. 4, 2133 (2013).

16. Lee, E. K. et al. Chemically robust ambipolar organic transistor array directly patterned by photolithography. Adv. Mater. 29, 1605282 (2017).

17. $\mathrm{Hu}, \mathrm{X}$. et al. Directly photopatternable polythiophene as dual-tone photoresist. Macromolecules 50, 7258-7267 (2017).

18. Jacobs, I. E. et al. Direct-write optical patterning of P3HT films beyond the diffraction limit. Adv. Mater. 29, 1603221 (2017).

19. DeFranco, J. A., Schmidt, B. S., Lipson, M. \& Malliaras, G. G. Photolithographic patterning of organic electronic materials. Org. Electron. 7, 22-28 (2006).

20. Baeg, K.-J., Facchetti, A. \& Noh, Y.-Y. Effects of gate dielectrics and their solvents on characteristics of solution-processed $n$-channel polymer fieldeffect transistors. J. Mater. Chem. 22, 21138-21143 (2012).

21. Minemawari, H. et al. Inkjet printing of single-crystal films. Nature 475, 364-367 (2011).

22. Singh, M., Haverinen, H. M., Dhagat, P. \& Jabbour, G. E. Inkjet printingprocess and its applications. Adv. Mater. 22, 673-685 (2010).

23. Guo, L. J. Nanoimprint lithography: methods and material requirements. $A d v$. Mater. 19, 495-513 (2007).

24. Tsiminis, G. et al. Nanoimprinted organic semiconductor laser pumped by a light-emitting diode. Adv. Mater. 25, 2826-2830 (2013).

25. Hermerschmidt, F., Choulis, S. A. \& List-Kratochvil, E. J. W. Implementing inkjet-printed transparent conductive electrodes in solution-processed organic electronics. Adv. Mater. Technol. 4, 1800474 (2019).

26. Sirringhaus, $\mathrm{H}$. et al. High-resolution inkjet printing of all-polymer transistor circuits. Science 290, 2123-2126 (2000).

27. Kahle, F. J., Saller, C., Köhler, A. \& Strohriegl, P. Crosslinked semiconductor polymers for photovoltaic applications. Adv. Energy Mater. 7, 1700306 (2017).

28. Rumer, J. W. \& McCulloch, I. Organic photovoltaics: crosslinking for optimal morphology and stability. Mater. Today 18, 425-435 (2015).

29. Reichmanis, E. \& Thompson, L. F. Polymer materials for microlithography. Chem. Rev. 89, 1273-1289 (1989).

30. Keivanidis, P. E., Khong, S.-H., Ho, P. K. H., Greenham, N. C. \& Friend, R. H. All-solution based device engineering of multilayer polymeric photodiodes: minimizing dark current. Appl. Phys. Lett. 94, 173303 (2009).

31. Tao, C. et al. Controlling hierarchy in solution-processed polymer solar cells based on crosslinked P3HT. Adv. Energy Mater. 3, 105-112 (2013).

32. Rumer, J. W. et al. Dual function additives: a small molecule crosslinker for enhanced efficiency and stability in organic solar cells. Adv. Energy Mater. 5, 1401426 (2015).

33. Khong, S.-H. et al. General photo-patterning of polyelectrolyte thin films via efficient ionic bis (fluorinated phenyl azide) photo-crosslinkers and their postdeposition modification. Adv. Funct. Mater. 17, 2490-2499 (2007).

34. Liu, B. et al. High internal quantum efficiency in fullerene solar cells based on crosslinked polymer donor networks. Nat. Commun. 3, 1321 (2012). 
35. Png, R.-Q. et al. High-performance polymer semiconducting heterostructure devices by nitrene-mediated photocrosslinking of alkyl side chains. Nat. Mater. 9, 152 (2010).

36. Cho, N. et al. In-situ crosslinking and n-doping of semiconducting polymers and their application as efficient electron-transporting materials in inverted polymer solar cells. Adv. Energy Mater. 1, 1148-1153 (2011).

37. Kim, B. J., Miyamoto, Y., Ma, B. \& Frechet, J. M. J. Photocrosslinkable polythiophenes for efficient, thermally stable, organic photovoltaics. $A d v$. Funct. Mater. 19, 2273-2281 (2009).

38. Cai, S. X., Glenn, D. J., Kanskar, M., Wybourne, M. N. \& Keana, J. F. W. Development of highly efficient deep-UV and electron beam mediated crosslinkers: synthesis and photolysis of bis (perfluorophenyl) azides. Chem. Mater. 6, 1822-1829 (1994).

39. Bräse, S., Gil, C., Knepper, K. \& Zimmermann, V. Organic azides: an exploding diversity of a unique class of compounds. Angew. Chem. Int. Ed. 44, 5188-5240 (2005).

40. Rodriquez, D. et al. Comparison of methods for determining the mechanical properties of semiconducting polymer films for stretchable electronics. ACS Appl. Mater. Interfaces 9, 8855-8862 (2017).

41. Kim, J.-H. et al. Tensile testing of ultra-thin films on water surface. Nat. Commun. 4, 2520 (2013).

42. Zaumseil, J. \& Sirringhaus, H. Electron and ambipolar transport in organic field-effect transistors. Chem. Rev. 107, 1296-1323 (2007).

43. Kang, M. S. \& Frisbie, C. D. A pedagogical perspective on ambipolar FETs. ChemPhysChem 14, 1547-1552 (2013).

44. Choi, H. H., Cho, K., Frisbie, C. D., Sirringhaus, H. \& Podzorov, V. Critical assessment of charge mobility extraction in fets. Nat. Mater. 17, 2-7 (2017).

45. Noriega, R. et al. A general relationship between disorder, aggregation and charge transport in conjugated polymers. Nat. Mater. 12, 1038-1044 (2013).

46. Stucchi, E., Dell'Erba, G., Colpani, P., Kim, Y. H. \& Caironi, M. Low-voltage, printed, all-polymer integrated circuits employing a low-leakage and highyield polymer dielectric. Adv. Electron. Mater. 4, 1800340 (2018).

47. Kwon, J. et al. Three-dimensional monolithic integration in flexible printed organic transistors. Nat. Commun. 10, 54 (2019).

\section{Acknowledgements}

This work was supported by the Samsung Research Funding \& Incubation Center of Samsung Electronics under project number SRFC-MA1901-05.

\section{Author contributions}

M.S.K., B.K., J.H.C. conceived the concept, designed the experiments, and supervised the work. M.J.K. and M.L. carried out most of the experimental work and data analyses M.J.K. fabricated devices. M.L. synthesized the materials. H.M., S.K., J.Y., W.L., and H.K. characterized films. D.Y.R., D.H.K., and J.H.C. assisted with the data analyses. All authors discussed the progress of research and contributed to editing the paper.

\section{Competing interests}

The authors declare no competing interests.

\section{Additional information}

Supplementary information is available for this paper at https://doi.org/10.1038/s41467020-15181-4.

Correspondence and requests for materials should be addressed to M.S.K., B.K. or J.H.C.

Peer review information Nature Communications thanks the anonymous reviewers for their contribution to the peer review of this work.

Reprints and permission information is available at http://www.nature.com/reprints

Publisher's note Springer Nature remains neutral with regard to jurisdictional claims in published maps and institutional affiliations.

\begin{abstract}
(c) (i) Open Access This article is licensed under a Creative Commons Attribution 4.0 International License, which permits use, sharing, adaptation, distribution and reproduction in any medium or format, as long as you give appropriate credit to the original author(s) and the source, provide a link to the Creative Commons license, and indicate if changes were made. The images or other third party material in this article are included in the article's Creative Commons license, unless indicated otherwise in a credit line to the material. If material is not included in the article's Creative Commons license and your intended use is not permitted by statutory regulation or exceeds the permitted use, you will need to obtain permission directly from the copyright holder. To view a copy of this license, visit http://creativecommons.org/ licenses/by/4.0/.
\end{abstract}

(C) The Author(s) 2020 